Revista Mexicana de Economía y Finanzas Nueva Época Volumen 14 Número 4, Octubre - Diciembre 2019, pp. 617-631 DOI: https://doi.org/10.21919/remef.v14i4.322

\title{
Efectos de la comunicación sobre los errores de pronóstico de inflación: evidencia para Colombia para el período 2008-2016
}

\author{
Juan Camilo Anzoátegui Zapata ${ }^{1}$ \\ Universidad Autónoma Latinoamericana, Colombia \\ Juan Camilo Galvis Ciro ${ }^{2}$ \\ Universidad Pontificia Bolivariana, Colombia \\ (Recepción: 2/agosto/2018, aceptado: 22/enero/2019)
}

\section{Resumen}

El objetivo de este trabajo es examinar los efectos de la comunicación del banco central sobre los errores de pronóstico de inflación para Colombia en el período 2008 - 2016. La metodología empleada consiste en un análisis econométrico compuesto por: i) La estimación de un modelo EGARCH; ii) El uso de Vector Auto regresivos (VAR); y iii) Un análisis de descomposición de varianza. Los resultados del trabajo muestran que los anuncios de política monetaria generan efectos importantes sobre los errores de pronóstico. La principal recomendación de política es que la comunicación es una herramienta que tiene el banco central para reducir los errores de pronóstico. En particular, la comunicación tiene el potencial para lograr una convergencia entre las expectativas de inflación y los objetivos perseguidos por el banco central. La principal limitación está relacionada con la falta de información más desagregada sobre las expectativas de inflación que no permiten analizar su reacción ante diferentes noticias macroeconómicas. La originalidad del trabajo consiste en analizar los errores de pronóstico de inflación con base en la inflación compensada de los títulos de deuda pública.

Clasificación JEL: E52, E58, E63

Palabras clave: Comunicación del banco central, errores de pronóstico, política monetaria

\section{The Communication Effects on Inflation Forecast Errors: Empirical Evidence from Colombia for the period 2008-2016}

\section{Abstract}

This paper aims to examine the effects of the central bank's communication on inflation forecasting errors for Colombia in the 2008-2016 period. The empirical evidence is composed of: i) The estimation of an EGARCH model, ii) useof VAR models; and iii) variance decomposition analysis. The results show that monetary policy announcements generate important effects on forecasting errors. The main policy recommendation is that central bank communication is a monetary policy tool to reduce forecast errors. In particular, communication has the potential to achieve a convergence between inflation expectations and inflation target. The main limitation is related to the lack of more disaggregated information on inflation expectations. This does not allow analyzing their reaction to different macroeconomic news. The originality of the paper consists in analyzing the inflation forecast errors based on the compensated inflation of the public debt securities.

JEL Classification: E52, E58, E63

Keywords: Central Bank Communication, Forecasting errors, Monetary Policy

\footnotetext{
${ }^{1}$ Dirección: Carrera 55a , No. 49-51, Medellín - Colombia, Tel: 5112199 ext 104, E-mail: juan.anzoategui@unaula.edu.co

${ }^{2}$ E-mail: jcgalvisciro@gmail.com
} 
REMEF (The Mexican Journal of Economics and Finance)

Efectos de la comunicación sobre los errores de pronóstico de

\section{Introducción}

La gestión de las expectativas de inflación es fundamental para consolidar la credibilidad de la política monetaria y conseguir un mejor desempeño macroeconómico (Johnson, 2003; Ehrmann et al., 2011; Scharnagl y Stapf, 2015). En medio de un régimen de metas de inflación, el banco central cuenta con diferentes herramientas que permiten incrementar la transparencia y ayudan a reducir las asimetrías de información en el mercado financiero. Como resultado, los pronósticos de inflación del mercado tienden a ser acertados (Bernanke et al., 1999; Geraats, 2002; Demertzis et al.2009; Crowe, 2010).

Debido a la importancia que tiene la percepción de los agentes sobre la política económica, existen varios estudios que evalúan la influencia de bancos centrales en el anclaje de las expectativas. Una parte de la literatura se enfoca en los desacuerdos del pronóstico de inflación entre los agentes (ver, por ejemplo, Mankiw et al., 2004; Capistrán y Timmermann, 2009; Patton y Timmermann 2010; Ehrmann, Eijffinger, y Fratzscher, 2012; Dovern, Fritsche y Slacalek; 2009). Otra parte de la literatura evalúa las encuestas de inflación y su capacidad de pronóstico. Esta literatura es reciente y muestra que las metas de inflación, las sorpresas de política monetaria, el ciclo económico y los pronósticos del banco central impactan los errores de pronóstico de inflación (Johnson, 2003; Kurz, 2005; Siklos, 2008; Dräger y Lamla, 2012; Pedersen, 2015; Tovar, 2017 y Apergis, 2017).

A pesar de estas evidencias empíricas para gestionar mejor las expectativas, en las economías emergentes los errores en los pronósticos de inflación son diferentes de cero en términos estadísticos como consecuencia de la persistencia en los ciclos económicos (Tovar, 2017). Además de esto, los agentes son ineficientes en el uso de la información disponible para realizar y actualizar sus pronósticos. En el mismo contexto, Pedersen (2015) y Apergis (2017) señalan que los cambios y los errores en los pronósticos de inflación entregados al público por parte de los bancos centrales en economías emergentes, afectan los pronósticos de inflación de los agentes privados. Frente a estas evidencias, este estudio amplía el análisis sobre los errores de pronósticos y coloca énfasis en ciertos factores que afectan la formación de expectativas de inflación en las economías emergentes. En particular, toma en cuenta las sorpresas de política monetaria, la volatilidad del precio de las materias primas y la volatilidad externa como factores que afectan los pronósticos de inflación.

En este documento, se utilizan expectativas de inflación para construir el error de pronóstico de inflación de tal forma que éste sea una medida asociada a la credibilidad de la política monetaria. Hasta nuestro conocimiento, no existen estudios que investiguen los efectos de la comunicación en los errores de pronóstico con base en las expectativas de inflación implícitas en los bonos de deuda pública para economías emergentes. Por tanto, el presente documento busca llenar ese vacío en la literatura.

El principal objetivo del presente estudio es analizar el efecto de la comunicación de la política monetaria sobre el anclaje de las expectativas de inflación para la economía colombiana en el período posterior a la adopción del régimen de metas de inflación. La hipótesis principal del trabajo consiste en que el Banco Central de Colombia, mediante la comunicación de sus decisiones sobre la tasa de política monetaria, afecta el error de pronóstico de inflación de los agentes del mercado financiero de la economía colombiana. En concreto, se espera que los comunicados de prensa tengan la capacidad de anclar las expectativas en la meta de inflación.

La importancia de la hipótesis postulada radicada, de forma principal, en la relevancia de ampliar el estudio sobre los efectos de la comunicación en el control de las expectativas del mercado financiero en economías emergentes. Este documento contribuye a entender la forma en que la comunicación del banco central afecta los errores de pronóstico de inflación y aporta a la literatura en varios aspectos. En primer lugar, conforme la metodología aplicada por Mankiw et al.(2004) y Pedersen (2015), muestra que los anuncios de política 
monetaria generan efectos importantes sobre los errores de pronóstico. En segundo lugar, analiza como estudio de caso la economía colombiana, una economía que tiene una brecha en la literatura sobre los posibles efectos de la comunicación en el control de las expectativas de inflación del mercado financiero. En tercer lugar, evidencia que la comunicación del banco tiene el potencial para disminuir los errores de pronóstico de inflación. En suma, los resultados encontrados permiten a los administradores de la política monetaria desarrollar estrategias más eficientes para el anclaje de las expectativas y la consolidación de la credibilidad del banco central.

La elección de Colombia como caso de estudio se debe a que es una economía emergente que adoptó la inflación objetivo desde el año 2000 y ha perfeccionado los canales de comunicación del banco central con el público. Además de esto, cuenta con grado de inversión y el desarrollo del mercado de capitales se fundamenta en el auge del mercado de deuda pública interna. En suma, Colombia representa un caso interesante para analizar la conducción de las expectativas de inflación.

Además de esta introducción, el resto del documento está organizado como sigue. En la segunda sección, es definido y analizado el error de pronóstico de inflación y es presentada la metodología para medir el efecto de la comunicación sobre el error de pronóstico. En la tercera sección, son realizadas las estimaciones econométricas y se discuten los resultados obtenidos. Por último, son presentadas las conclusiones.

\section{Metodología}

El anclaje de las expectativas de inflación en las metas anunciadas es fundamental para el banco central porque indica el nivel de confianza del público y provee información útil sobre la credibilidad de la política monetaria (Gürkaynak, Levin, y Swanson, 2010; Levin, 2014). Los errores de pronóstico de inflación reflejan desacuerdos de los agentes con respecto a la postura de la política monetaria. En consecuencia, la reducción de los errores de pronóstico del mercado contribuye para el control de la inflación observada y aporta solidez al sistema financiero (Mankiw, Reis y Wolfers 2004).

Para la disminución del sesgo de pronóstico, en un régimen de metas de inflación la transparencia y la comunicación del banco central son herramientas importantes debido a que permiten una convergencia de las expectativas de inflación hacia la meta (Ehrmann, Eijffinger y Fratzscher, 2012). En el caso de economías emergentes existen varias fuentes de incertidumbre y choques externos que dificultan el anclaje de las expectativas en los objetivos de política monetaria (De Pooter et al., 2014). En particular, las decisiones del banco central en economías emergentes son difíciles de anticipar y contienen sorpresas que pueden desalinear las expectativas de inflación (Ellingsen y Söderström, 2001).

Para entender de qué forma responden los agentes ante las decisiones del banco central, se puede hacer uso de las estimaciones de expectativas de mercado las cuales han demostrado ser mejores predictores que las estimaciones basadas en encuestas. Las medidas derivadas de los precios de los activos tienen una disponibilidad diaria y revelan el pronóstico de las expectativas de inflación realizadas por los inversionistas (Christensen, Dion y Reid, 2004; Gürkaynak et at., 2005; Söderlind, 2011).

La medida de mercado más utilizada para estimar las expectativas de inflación es el Break Even Inflation (de ahora en adelante, BEI), también llamada inflación compensada (Gürkaynak et al., 2010 y Söderlind, 2010). La compensación por inflación captura la compensación que los inversionistas demandan por la inflación esperada y por los riesgos de incertidumbre asociados a la inflación. Por consiguiente, la BEI es una medida de las expectativas de inflación basada en los mercados financieros y se calcula como la tasa de inflación que hace indiferente a un inversionista entre los bonos nominales y los bonos indexados a inflación. Es decir,

$$
\pi_{t}^{e}=f_{t, \text { nom }^{m}}^{m}-f_{t, \text { real }}^{m}
$$


Donde $\pi_{t}^{e}$ son las expectativas de inflación, $f_{t, \text { nom }}{ }^{m}$ es el rendimiento del título nominal, $f_{t, \text { real }}{ }^{m}$ es el rendimiento del título indexado y $\mathrm{m}$ es el período de maduración del título. En el caso colombiano, la BEI ha mostrado ser una medida eficiente de las expectativas de inflación a corto plazo (Melo y Granados, 2010). Por tanto, en este trabajo son utilizados títulos con madurez a 1 año para calcular las expectativas de inflación a 1 año (12 meses).

Una vez se construye una serie de expectativas de inflación, existen varias formas de medir el error de pronóstico. De acuerdo con la metodología de Mankiw, Reis y Wolfers (2004), para medir el error de pronóstico fueron utilizadas las expectativas de inflación como una medida del pronóstico de inflación. El error es calculado como la diferencia entre la meta de inflación anunciada por el banco central y el pronóstico de inflación. Se asume que la meta de inflación del banco central es una expectativa que tiene el banco sobre el comportamiento de la inflación para final de cada año. Por esto, la diferencia entre las expectativas de inflación de los agentes del mercado con respecto a la meta del banco central se tomó como un error en el pronóstico de inflación para la economía colombiana. $^{3}$ Son considerados como errores tanto las desviaciones positivas y negativas de la meta. Por tanto, el error de pronóstico es construido como el valor absoluto de la diferencia entre la meta de inflación y las expectativas de inflación. Esto es:

$$
\varepsilon_{t}=\left|\pi^{*}-\pi_{t}^{e}\right|
$$

Con base en datos mensuales, el comportamiento en el tiempo de las expectativas de inflación (Break-Even Inflation rates) y la meta de inflación son presentadas en la figura 1.

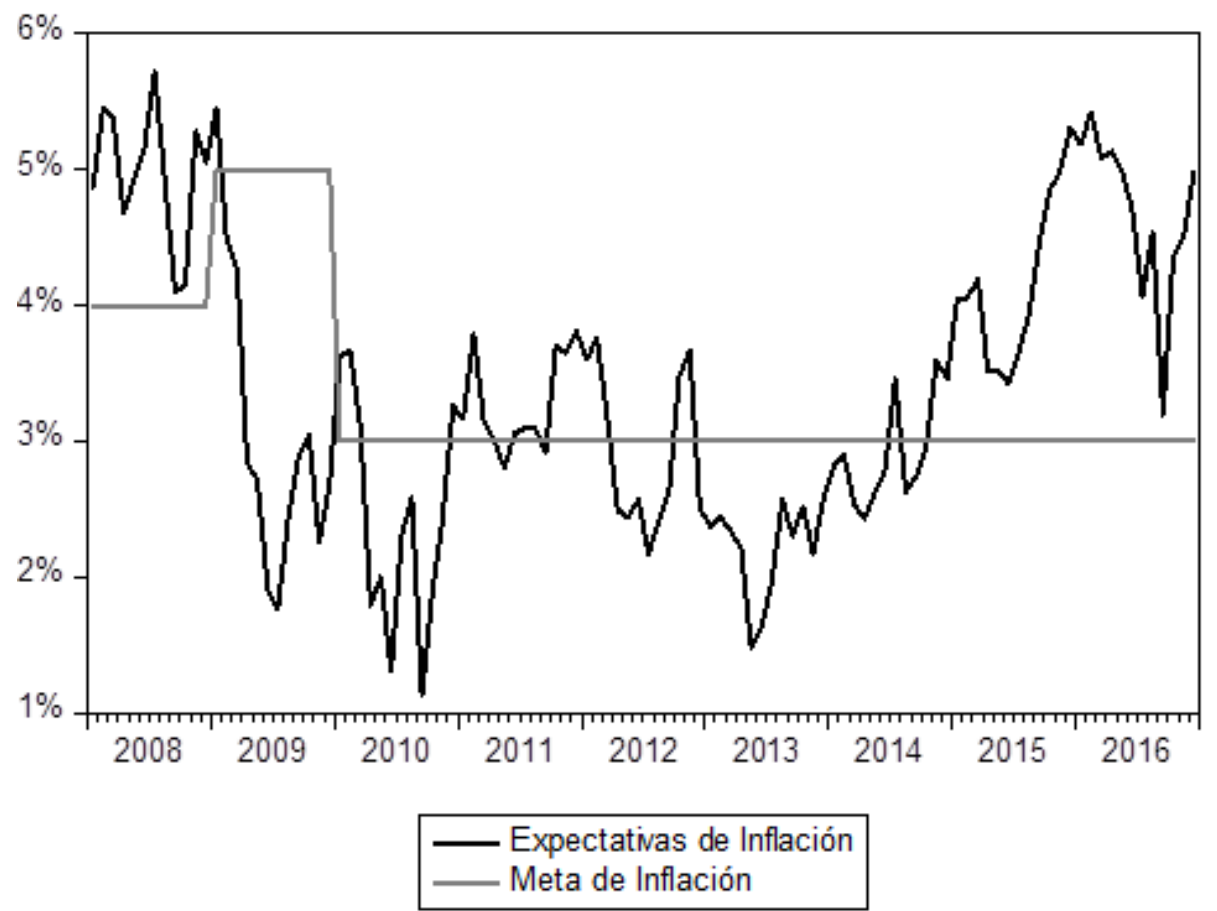

Figura 1. Expectativas de inflación y meta de inflación Nota: Elaboración propia. Datos obtenidos del Banco Central de Colombia.

\footnotetext{
${ }^{3}$ Debido a que la meta de inflación del Banco Central de Colombia es una meta anual (a 12 meses), deben ser utilizadas expectativas de inflación a 1 año (12 meses) para calcular el error de pronóstico con el fin de que sea un error construido sobre variables equiparables. Es por esta razón que sólo fueron utilizados los títulos públicos con madures a 1 año.
} 
A partir de las variables presentes en la figura 1, se presenta a continuación el comportamiento de los errores de pronóstico para la economía colombiana durante el período comprendido entre 2008 - 2016 (ver figura 2). ${ }^{4}$ Un error positivo denota una divergencia en las expectativas de inflación con relación a la meta establecida por el banco central y un error cercano a cero denota convergencia entre las expectativas y la meta del banco central, es decir, credibilidad.

En el caso colombiano, se observa que el error de pronóstico fue en promedio de $0.096 \%$ (ver figura 2). Con una meta puntual de inflación variable en los primeros años, se puede afirmar que el comportamiento observado implica dos cosas. Primero, en los períodos donde la meta central de inflación fue mayor, el error de pronóstico fue más alto. Segundo, las expectativas de inflación presentan un proceso de convergencia hacia la meta central de inflación cuando aquella fue fijada en $3 \%$ (desde el 2010 en adelante). No obstante, el error aumentó de forma considerable entre mitad de 2015 y finales de 2016 cuando las expectativas de inflación se aceleraron.

Por medio del comportamiento observado en la figura 2, es posible destacar tres períodos de desanclaje de las expectativas de inflación para el período analizado. Un primer período en el 2008-2010 donde el error de pronóstico fue en promedio de $1.2736 \%$. Un segundo período entre 2011-2014 donde el error de pronóstico cayó y fue en promedio de $0.5008 \%$. Finalmente, un tercer período entre finales de 2014 y 2016 donde las expectativas de inflación aumentaron y, como resultado, el error de pronóstico fue en promedio de 1.4216

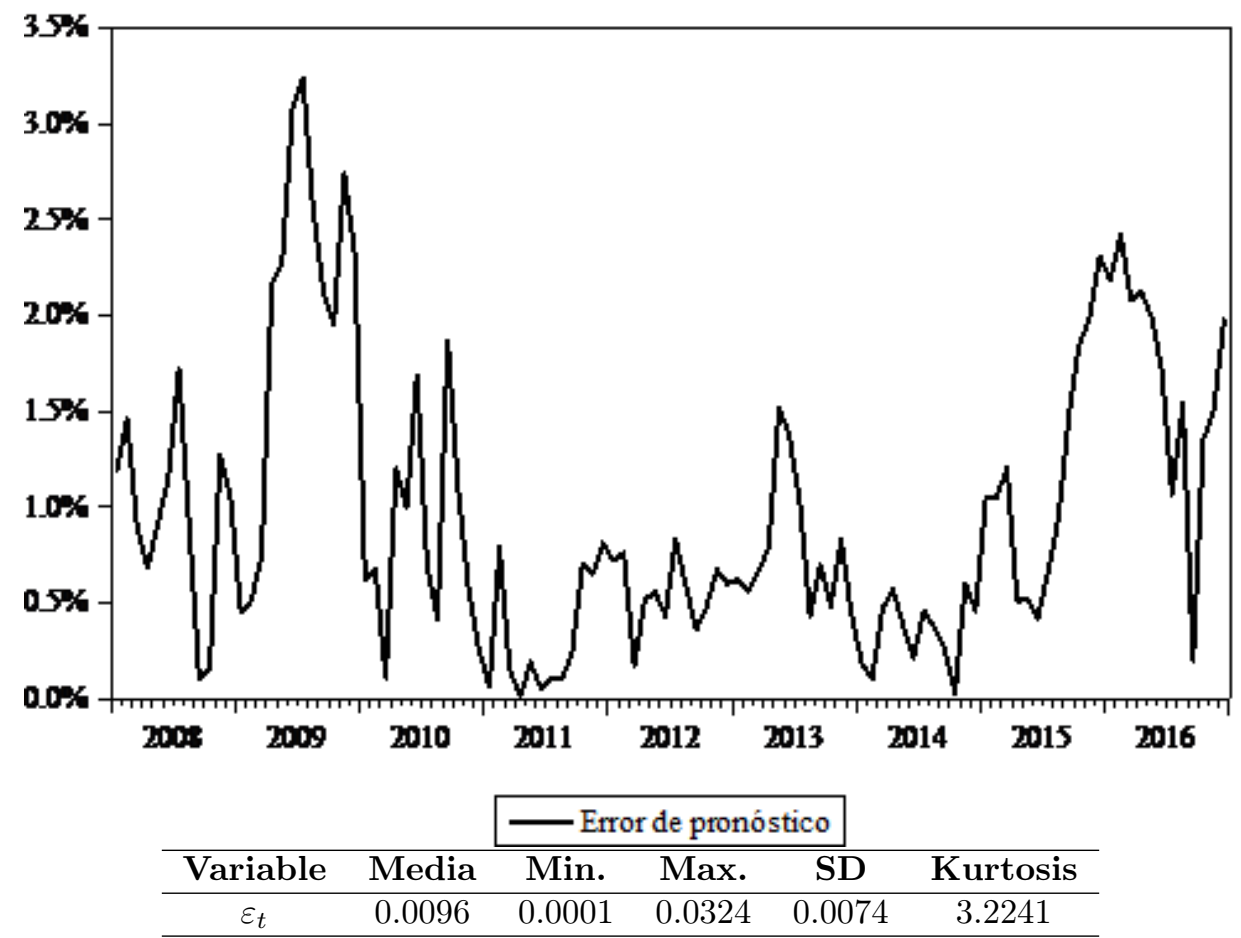

Figura 2. Evolución del error de pronóstico para Colombia

Nota: Elaboración propia. Datos obtenidos del Banco Central de Colombia.

Los errores de pronóstico adquieren relevancia en un régimen de metas de inflación debido a que la comunicación más sistemática del banco central lleva a que el público comprenda mejor la forma en que se toman las decisiones de política monetaria. En

\footnotetext{
${ }^{4}$ La elección del período inicial se debe a que la información sobre los bonos indexados en Colombia sólo está disponible desde el año 2008.
} 
especial, los objetivos de inflación llevan a que los mercados formulen expectativas sobre las próximas decisiones de los formuladores de política (Connolly y Kohler, 2004; Blinder et al., 2008).

Por lo general, existen componentes esperados e inesperados en las decisiones del banco central. El componente esperado ya ha sido incorporado en las expectativas de los agentes y no tiene grandes efectos en los mercados (Blinder et al., 2008). En consecuencia, para analizar los efectos de la comunicación en la toma de decisiones es necesario colocar especial atención al componente no anticipado presente en cada decisión de política monetaria (Jansen y De Haan 2007; Gürkaynak, et al., 2010; Ehrmann et al., 2011).

El componente no anticipado es, como tal, una sorpresa de política monetaria presente en la comunicación del banco central y se origina cuando el mercado financiero no predice el comportamiento de la tasa de interés porque no comprenden la dirección o postura de la política monetaria. El componente no anticipado está asociado a la información asimétrica en poder del banco central que el público no conocía. En consecuencia, las sorpresas generan cierto ruido en el mercado financiero y afectan el anclaje de las expectativas de inflación en la meta de inflación, es decir; la comunicación tiene impacto en los errores de pronóstico (Filardo y Guinigundo, 2008 y Montes et al., 2016).

Para efectos estadísticos, conforme a la metodología propuesta por Kuttner (2001) y Connolly y Kohler (2004), la sorpresa de política monetaria se estima como la variación de la tasa de interés del mercado en los días en que el banco central realiza un anuncio hacia el mercado financiero. Las tasas de interés a un mes reflejan las expectativas del mercado sobre las decisiones de política monetaria (Galvis, De Moraes y Anzoátegui, 2017). Es decir, estas tasas reaccionan en relación a las diferencias en las expectativas de los agentes y las decisiones esperadas por el banco central (ver, por ejemplo, Kuttner, 2001). En el caso colombiano, el componente no anticipado de política monetaria puede ser calculado por medio de la variación de la tasa IBR - Indicador Bancario de Referencia - una tasa de interés del mercado financiero colombiano que permite calcular las decisiones de política inesperadas (Galvis y Anzoátegui, 2017).

El IBR refleja el precio al que los bancos están dispuestos a ofrecer o a captar recursos entre sí y como tal, es consecuencia de las cotizaciones de recursos en el mercado financiero colombiano. En específico, el IBR es una mediana de dichas cotizaciones de liquidez y, por tanto, se convierte en una tasa de interés representativa del mercado financiero colombiano y en aquella tasa donde se materializa el mecanismo de transmisión de la política monetaria (Galvis et al., 2017). ${ }^{5}$ Para construir las sorpresas en la comunicación del Banco Central de Colombia es calculada la variación de la tasa IBR. Es decir,

$$
S P M_{t}=I B R_{t}-I B R_{t-1}
$$

Siendo $S P M_{t}$ el componente inesperado en los anuncios de política monetaria en el día t, y el $I B R_{t}$ es el indicador bancario de referencia en el día t.

Para calcular la sorpresa de política monetaria, este estudio analiza los comunicados de prensa. Este canal de comunicación es un documento con periodicidad mensual y su propósito es dar a conocer las decisiones sobre las variaciones de la tasa de interés de política que ha tomado la Junta Directiva del Banco Central. Es un medio que recibe gran atención del público y permite identificar cambios en la política monetaria de manera oportuna. La información suministrada al público es concreta y su principal objetivo es comunicar de forma breve las razones clave atrás de cada decisión tomada sobre la tasa de interés de política.

\footnotetext{
${ }^{5}$ El IBR se publica desde 2008 como un indicador que puede medir en tiempo real las condiciones de liquidez de corto plazo del mercado monetario. La elección de la tasa de interés cotizada por los ocho bancos principales del mercado financiero es un hecho especulativo. Esto significa que depende de las expectativas de corto plazo del comportamiento de la tasa de interés de política y de las condiciones actuales de liquidez de cada participante.
} 
Dado el método apuntado antes para medir la sorpresa monetaria, la variación de la tasa IBR entre los días en que es emitido el comunicado de prensa, es presentada en la figura 3. El componente inesperado de política monetaria se construye como la diferencia entre la tasa overnight el día en que el Banco Central se comunicó con el mercado financiero (día t) con relación a la tasa que existía el día anterior de la comunicación del banco central (día t-1). Los días en que el banco central anuncia sus decisiones fueron establecidas con base en el calendario publicado por la Junta Directiva del Banco Central. Cuando el comunicado de prensa es anunciado después del cierre de la bolsa de valores de Colombia, se fijó el día del comunicado para el siguiente día hábil. Acorde a la figura 3, la variación de la tasa IBR muestra que existe una reacción del mercado frente a los comunicados de prensa, lo que permite identificar componentes inesperados y efectos importantes sobre el mercado financiero una vez se ha realizado el anuncio por parte del banco central.

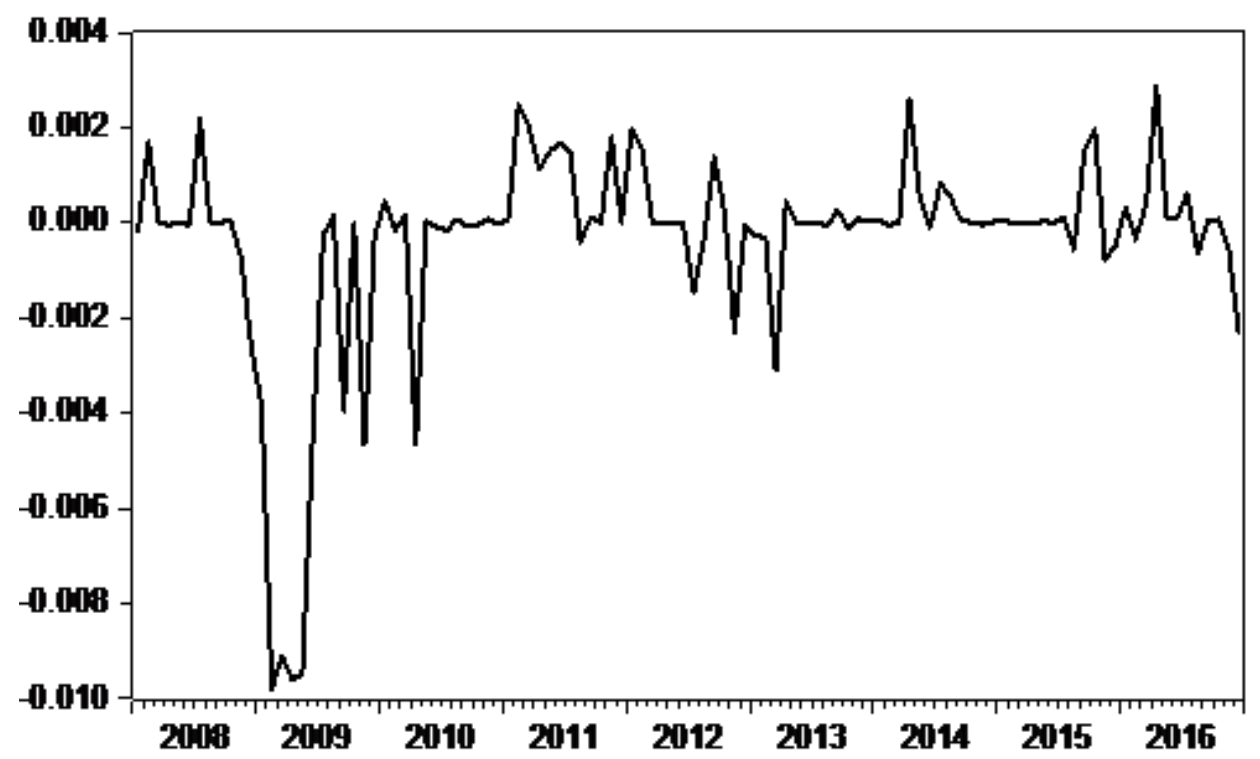

Figura 3. Componente inesperado de política monetaria

Fuente: Elaboración propia. Datos obtenidos del Banco Central de Colombia.

Según se observa en la figura 3, los cambios en la tasa IBR durante los días de la comunicación del banco implican divergencias en las expectativas del mercado y afectan los pronósticos sobre las futuras intenciones en la conducción de política monetaria. A su vez, variaciones inesperadas de la tasa de política llevan a que los inversionistas revaloricen los rendimientos esperados en los bonos nominales e indexados. Es decir, cuando el mercado financiero reacciona ante las decisiones de política monetaria es de esperarse una recomposición de las expectativas de inflación con relación a los objetivos perseguidos por el banco central. Conforme muestran Mankiw, Reis y Wolfers (2004), también es importante destacar que el error de pronóstico muestra una inercia importante en el tiempo. Dado esto, el modelo básico considerado en este estudio es el siguiente:

$$
\varepsilon_{t}=\alpha_{0}+\alpha_{1} \varepsilon_{t-1}+\alpha_{2} S P M_{t}+\alpha_{3} \sum_{i=1}^{n} X_{i}+u_{t}
$$

Donde $\varepsilon_{t}$ es el error de pronóstico y $\varepsilon_{t-1}$ es el error de pronóstico del período anterior y captura la inercia en el error de pronóstico. El término SPM es el componente sorpresa de la política monetaria y $X_{i}$ es un vector de variables explicativas o de control. 
El término error es $u_{t}=v_{t} h_{t} \sim\left(0, h_{t}^{2}\right)$, con $v_{t}=\frac{u_{t}}{h_{t}} \sim i i d(0,1)$. Es asumido que el término error tiene una varianza condicional que sigue el siguiente proceso:

$$
\log \left(h_{t}^{2}\right)=\varpi+\sum_{j=1}^{q} \beta_{j} \log \left(h_{t-j}^{2}\right)+\sum_{i=1}^{p} \alpha_{i}\left|\frac{u_{t-i}}{h_{t-i}}\right|+\sum_{k=1}^{r} \gamma_{k} \frac{u_{t-k}}{h_{t-k}}
$$

La ecuación [4] es estimada a través de un modelo EGARCH (heteroscedasticidad condicional autorregresiva generalizada exponencial). Engle (1982), determina que los modelos GARCH son más apropiados para analizar el mercado financiero, dado que las series financieras se caracterizan por la no normalidad de los rendimientos, las cuales se caracterizan porque no tienen distribución normal y muestran una curtosis alta. De acuerdo con Nelson (1991) esta especificación permite identificar de forma adecuada los cambios en la volatilidad condicional del error de pronóstico de inflación a choques positivos o negativos asociados a los anuncios de política del banco central y los correspondientes a las variables de control. Por lo tanto, la especificación elegida es un EGARCH $(1,1)$.

El vector de variables control contiene cuatro variables. Conforme señalan Mankiw et al.(2004) y Apergis (2017), las variaciones de los errores de pronóstico también son afectadas por otras variables no relacionadas con las decisiones de política monetaria. Existe evidencia que muestra que las variaciones de la tasa de cambio afectan las expectativas de inflación en la economía colombiana (Rincón y Rodríguez, 2016). Así, una primera variable de control a considerar es la variación de la tasa de cambio nominal $(\Delta e)$.

Los riesgos asociados a la incertidumbre del mercado financiero global es un factor clave en los flujos de capital de las economías emergentes dado que cambios en las expectativas sobre el mercado internacional propician variaciones en el precio de los activos y, por lo tanto, en la inflación compensada (Byrne y Fiess, 2016). Un indicador que mide el apetito de los inversionistas por el riesgo es medido por el índice de mercado S\&P 500 o índice VIX, el cual señaliza el riesgo global de los flujos de capital. Dado esto, como una segunda variable de control es utilizada la variación del índice VIX ( $\Delta$ VIX).

Una tercera variable está asociada a la situación de la liquidez mundial, un indicador de referencia para todo inversionista. La tasa de interés de los bonos de los Estados Unidos refleja las condiciones económicas globales y funciona como un proxy de la tasa de interés libre de riesgo. Además, la tasa de interés de los bonos del tesoro americano refleja el costo internacional del capital y afecta los rendimientos de los bonos soberanos de las economías domésticas (González-Rozada y Levy-Yeyati, 2008). En consecuencia, como otra variable control es utilizada la tasa de interés de los bonos del tesoro de los Estados Unidos $\left(\Delta \mathrm{i}^{*}\right)$.

De acuerdo con Alichi et al.(2011) en economías emergentes existe un passthrough importante de los precios del petróleo sobre los precios domésticos. Además, el precio del petróleo tiene fuerte incidencia en la estabilidad macroeconómica de la economía colombiana. En particular, la volatilidad del precio del petróleo influye en los rendimientos de los títulos públicos nominales e indexados y, por consiguiente, en la compensación de inflación (Martínez et al., 2015). De esta forma, como una cuarta variable control son utilizadas las variaciones del precio del petróleo $(\Delta \mathrm{OIL})$.

En suma, junto con las sorpresas de política monetaria presentes en la comunicación, se establecen cuatro variables control $\left(\Delta \mathrm{e}, \Delta \mathrm{VIX}, \Delta \mathrm{i}^{*}, \Delta \mathrm{OIL}\right)$. Estas variables control fueron utilizadas en desviación estándar porque ellas están medidas en diferentes unidades (pesos colombianos, dólares estadounidenses y números índices). Una forma de hacer comparables los coeficientes de regresión de las variables consiste en utilizar la desviación estándar de los datos y no sus variaciones. La desviación estándar fue calculada con los datos de cada serie entre el día t y el día $t+1$, siendo $t$ el día del comunicado de prensa del banco central. Esta es una metodología utilizada de forma recurrente en la literatura (ver, por ejemplo, Gürkaynak, et al., 2010; Ehrmann, et al., 2011; De Pooter et al., 2014). 
Los datos usados en este estudio corresponden al período que se extiende entre enero de 2008 hasta diciembre de 2016. La fuente y las estadísticas descriptivas de las variables se presentan a continuación en la tabla 1 y tabla 2 .

Tabla 1. Variables utilizadas y fuente de los datos

\begin{tabular}{|c|c|c|}
\hline Nombre de la variable & Descripción & Fuente \\
\hline$S P M$ & Indicador Bancario de Referencia (IBR) & $\begin{array}{c}\text { Banco Central de Colombia } \\
\text { http://www.banrep.gov.co/es/ibr }\end{array}$ \\
\hline$f_{\text {nominal }}$ & Títulos de deuda pública nominales & $\begin{array}{c}\text { Banco Central de Colombia } \\
\text { http://www.banrep.gov.co/es/tes }\end{array}$ \\
\hline$f_{\text {index }}$ & Títulos de deuda pública indexados & $\begin{array}{c}\text { Banco Central de Colombia } \\
\text { http://www.banrep.gov.co/es/tes }\end{array}$ \\
\hline$e$ & Tasa de cambio peso colombiano/dólar & $\begin{array}{c}\text { Banco Central de Colombia } \\
\text { http://www.banrep.gov.co/es/trm }\end{array}$ \\
\hline Oil & Cotización del barril de petróleo (WTI) & $\begin{array}{c}\text { Reserva Federal de St. Louis } \\
\text { (http://research.stlouisfed.org/) }\end{array}$ \\
\hline$V I X$ & $\begin{array}{l}\text { Îndice de volatilidad del Mercado de valores } \\
\qquad \text { S\&P } 500\end{array}$ & $\begin{array}{c}\text { Reserva Federal de St. Louis } \\
\text { (http://research.stlouisfed.org/) }\end{array}$ \\
\hline$i^{*}$ & $\begin{array}{l}\text { Tasa de interés de los títulos de deuda pública } \\
\text { del tesoro de los Estados Unidos a un mes }\end{array}$ & $\begin{array}{c}\text { Reserva Federal de St. Louis } \\
\text { (http://research.stlouisfed.org/) }\end{array}$ \\
\hline
\end{tabular}
Nota: Elaboración propia.

Tabla 2. Estadísticas descriptivas

\begin{tabular}{|c|c|c|c|c|c|}
\hline Variable & Media & Min. & Max. & Desviación st. & Curtosis \\
\hline spm & -0.0003 & -0.0098 & 0.0028 & 0.0022 & 11.2819 \\
\hline$\varepsilon_{t}$ & 0.0096 & 0.0001 & 0.0324 & 0.0074 & 3.2241 \\
\hline$\Delta e$ & 10.4868 & 0.0000 & 74.7910 & 12.2369 & 9.8898 \\
\hline$\Delta$ Oil & 0.9253 & 0.0100 & 10.4400 & 1.1505 & 44.6219 \\
\hline$\Delta i^{*}$ & 0.0112 & 0.0000 & 0.0848 & 0.0128 & 14.5510 \\
\hline$\Delta$ VIX & 0.8671 & 0.0000 & 8.9873 & 1.2403 & 21.0200 \\
\hline \multicolumn{7}{|c|}{ Nota: Elaboración propia. }
\end{tabular}

Antes de estimar la ecuación [4], es necesario adelantar los denominados test de raíz unitaria, Dickey-Fuller aumentado (ADF) y Phillips-Perron (PP) y un test de estacionariedad, Kwiatkowski-Phillips-Schmidt-Shin (KPSS), los cuales son presentados en la tabla 3. Los tests (ADF, PP) fueron analizados con un nivel de significancia de $1 \%$. En el caso del test KPSS, para que la serie sea definida estacionaria se comprobó significancia al $1 \%$. El objetivo es contrastar los resultados de los test ADF y PP con los resultados obtenidos del KPSS. Cuando al menos dos de los test indica que la serie no tiene raíz unitaria, entonces la serie es considerada estacionaria. De acuerdo a las estimaciones obtenidas, se puede afirmar que las variables a utilizar en la ecuación [4] son todas I(0).

Table 3. Tests de raíz unitaria y estacionariedad

\begin{tabular}{|c|c|c|c|c|c|c|c|c|c|c|c|c|}
\hline \multirow{2}{*}{ Series } & \multicolumn{4}{|c|}{ ADF } & \multicolumn{4}{|c|}{ PP } & \multicolumn{4}{|c|}{ KPSS } \\
\hline & Lags & Esp. & Test & C. V (1\%) & Band & Esp. & Test & C. V (1\%) & Band & Esp. & Test & C. V (1\%) \\
\hline spm & 0 & $\mathrm{~N}$ & -4.28 & -2.58 & 2 & $\mathrm{~N}$ & -4.16 & -2.58 & 7 & $\mathrm{C}, \mathrm{T}$ & 0.08 & 0.21 \\
\hline$\varepsilon_{t}$ & 0 & $\mathrm{C}$ & -3.58 & -3.49 & 1 & $\mathrm{C}$ & -3.53 & -3.49 & 8 & $\mathrm{C}$ & 0.16 & 0.73 \\
\hline$\Delta e$ & 1 & $\mathrm{C}, \mathrm{T}$ & -4.63 & -4.04 & 7 & $\mathrm{C}, \mathrm{T}$ & -8.69 & -4.04 & 8 & $\mathrm{C}, \mathrm{T}$ & 0.43 & 0.73 \\
\hline$\Delta$ Oil & 0 & $\mathrm{C}$ & -10.71 & -3.49 & 3 & $\mathrm{C}$ & -10.72 & -3.49 & 0 & C,T & 0.08 & 0.21 \\
\hline$\Delta i^{*}$ & 2 & $\mathrm{C}$ & -4.00 & -3.49 & 7 & $\mathrm{C}$ & -8.16 & -3.49 & 7 & $\mathrm{C}$ & 0.52 & 0.73 \\
\hline$\Delta$ VIX & 0 & $\mathrm{C}$ & -9.40 & -3.49 & 1 & $\mathrm{C}$ & -9.40 & -3.49 & 0 & $\mathrm{C}$ & 0.04 & 0.21 \\
\hline
\end{tabular}

Nota: Elaboración propia. C.V. = valor crítico. Tendencia $(\mathrm{T}), \mathrm{y} / \mathrm{o}$ constante $(\mathrm{C})$, o Ni tendencia ni Constante $(\mathrm{N})$ son incluidas con base en el criterio de información de Schwarz. El Test KPSS fue utilizado con banda Newey-West. 


\section{Estimaciones y resultados}

Los resultados de las estimaciones del modelo propuesto son presentados en la tabla 4. Primero es estimado el modelo básico que evalúa el efecto de la comunicación sobre los errores de pronóstico de inflación. Luego, se extiende el modelo base y se agrega cada variable de control al tiempo. De acuerdo con los resultados obtenidos, una primera inspección a los coeficientes asociados a la comunicación nos permite inferir que el signo encontrado está en línea con la perspectiva teórica esperada y los parámetros estimados son significativos (ver tabla 4).

Antes de interpretar los resultados, es importante apuntar que el banco central tiene una mejor y mayor información sobre el desempeño de la economía y cuando produce nuevas comunicaciones reduce la incertidumbre en el mercado financiero. Por tanto, cuando el banco central toma una decisión de política monetaria, el mercado ajusta su pronóstico ante el nuevo panorama esquematizado (Pedersen, 2015). Según se observa en la tabla 1, el coeficiente asociado a la comunicación de la política monetaria tiene efectos inversos y significativos en términos estadísticos sobre los errores de pronóstico en todos los modelos estimados. Con base en los resultados encontrados, es posible evidenciar que la comunicación disminuye del error de pronóstico, lo que permite inferir que el banco central tiene la capacidad de lograr una convergencia en las expectativas de inflación hacia la meta. Resultados similares son encontrados por Pedersen (2015), Dräger y Lamla (2012) y Johnson (2003).

Si se analizan los resultados de los otros parámetros, se observa que las variables financieras también ejercen efectos sobre los errores de pronóstico del mercado. En general, los signos de los coeficientes de estas variables están en línea con lo esperado y los coeficientes son significativos en los modelos estimados. El error de pronóstico muestra persistencia porque en muchos casos los agentes del mercado tardan en entender la coyuntura económica actual. Conforme muestran los resultados, las estimaciones arrojan que el rezago del error de pronóstico $\left(\varepsilon_{t-1}\right)$ es positivo y significativo al $1 \%$ en todos los modelos presentados. Es decir, la dinámica del rezago es importante y ayuda a explicar los errores de pronóstico del mercado financiero. Un resultado similar es encontrado en Mankiw, Reis y Wolfers (2004) y Babecký y Podpiera (2011).

Por otra parte, el parámetro asociado con la tasa de cambio $(\Delta e)$ es positivo y significativo. Los incrementos en la tasa de cambio tienen un pass-through importante sobre los precios internos en Colombia (Rincón y Rodríguez, 2016). Como resultado, aumentos en la tasa de cambio generan presiones en las expectativas de inflación y, como resultado, generan efectos en los errores de pronóstico. Resultados similares son encontrados en Siklos (2008), Pedersen (2015) y Apergis (2017).

También se observa que las estimaciones arrojadas por las variaciones del precio del petróleo $(\triangle O I L)$ muestran que el coeficiente es positivo y significativo al $1 \%$. En este contexto, es reforzada la evidencia empírica en la literatura que sugiere que los precios del petróleo influyen en los errores de pronóstico de inflación (ver, Pedersen, 2015; Bec y De Gaye, 2016). Por otra parte, el parámetro asociado con la tasa de interés externa $\left(i^{*}\right)$ es positivo y significativo en términos estadísticos. Ello sugiere que un incremento en la tasa de interés externa aumenta los errores de pronóstico por sus efectos potenciales en los movimientos de capitales y la tasa de cambio.

Por último, ya con relación al coeficiente del riesgo externo $(\Delta V I X)$ los resultados encontrados muestran que la evidencia está acorde con la literatura. El coeficiente es positivo, lo cual muestra que el riesgo global, medido a través del VIX tiene el potencial de mover los mercados financieros de las economías emergentes, afectar las expectativas y ejercer influencia sobre el error de pronóstico. Un resultado similar es obtenido en De Pooter et al., (2014). 
Tabla 4. Efectos de la comunicación sobre los errores de pronóstico $(\operatorname{EGARCH}(1,1))$

\begin{tabular}{|c|c|c|c|c|c|c|}
\hline Variable dep. & Modelo 1 & Modelo 2 & Modelo 3 & Modelo 4 & Modelo 5 & Modelo 6 \\
\hline \multirow{3}{*}{ Constante } & $0.0021^{* *}$ & 0.0013 & 0.0008 & $0.0011^{*}$ & $0.0009^{* * *}$ & $0.0003^{*}$ \\
\hline & -0.0009 & -0.001 & -0.0005 & -0.0006 & -0.0002 & -0.0001 \\
\hline & {$[2.2732]$} & [1.2724] & [1.6085] & {$[1.7452]$} & {$[3.7500]$} & {$[1.7476]$} \\
\hline \multirow{3}{*}{$\varepsilon_{t-1}$} & $0.7586^{* * *}$ & $0.7476^{* * *}$ & $0.8023^{* * *}$ & $0.7633^{* * *}$ & $0.8063^{* * *}$ & $0.7168 * * *$ \\
\hline & -0.0645 & -0.061 & -0.04 & -0.0533 & $-8.27 \mathrm{E}-05$ & -0.0002 \\
\hline & {$[11.7543]$} & [12.2445] & [20.0164] & [14.3164] & {$[9.7486]$} & [28.6912] \\
\hline \multirow{3}{*}{ MPS } & $-0.6289^{* * *}$ & $-0.5884^{* * *}$ & $-0.7038^{* * *}$ & $-0.6835^{* * *}$ & $-0.6380 * * *$ & $-0.7035^{* * *}$ \\
\hline & -0.1701 & -0.1377 & -0.12 & -0.1623 & -0.1144 & -0.0568 \\
\hline & {$[-3.6956]$} & {$[-4.2708]$} & {$[-5.8615]$} & {$[-4.2091]$} & {$[-5.525]$} & {$[-12.368]$} \\
\hline \multirow{3}{*}{$\Delta e$} & & $9.21 \mathrm{E}-05^{* *}$ & & & & $3.27 \mathrm{E}-05^{* *}$ \\
\hline & & $-4.21 \mathrm{E}-05$ & & & & $-1.55 \mathrm{E}-05$ \\
\hline & & [2.1881] & & & & [2.1134] \\
\hline \multirow{3}{*}{$\Delta O i l$} & & & $0.0007 * * *$ & & & $0.0009^{* * *}$ \\
\hline & & & -0.0003 & & & $-7.74 \mathrm{E}-05$ \\
\hline & & & [2.3895] & & & [12.638] \\
\hline \multirow{3}{*}{$\Delta i^{*}$} & & & & $0.0743^{* * *}$ & & $0.0297 * * *$ \\
\hline & & & & -0.0248 & & -0.0096 \\
\hline & & & & [2.9902] & & {$[3.0728]$} \\
\hline \multirow{3}{*}{$\Delta V I X$} & & & & & $0.0005^{* * *}$ & $0.0002 * * *$ \\
\hline & & & & & -0.0001 & $-6.55 \mathrm{E}-05$ \\
\hline & & & & & [3.3191] & {$[3.379]$} \\
\hline Log likelihood & 424.36 & 430.11 & 434.56 & 435.74 & 443.55 & 405.01 \\
\hline Obs. & 107 & 107 & 107 & 107 & 107 & 107 \\
\hline
\end{tabular}

Nota: Nivel de significancia: $(* * *)$ denota significancia a $0.01,\left({ }^{* *}\right)$ denota significancia a $0.05,\left(^{*}\right)$

denota significancia a 0.1. Desviación estándar entre paréntesis y estadística-t entre corchetes. 


\subsection{Análisis impulso respuesta y descomposición de varianza}

Con el fin de ampliar la evidencia de la comunicación del banco central sobre los errores de pronóstico de inflación, a continuación, se presenta un nuevo ejercicio empírico por medio de un modelo de Vectores Autorregresivos (VAR). Como es usual, la dinámica del análisis VAR es realizada por medio de funciones impulso-respuesta ya que estas permiten observar el efecto de un impulso en la comunicación causado por choques o innovaciones y sus efectos sobre los errores de pronóstico de inflación. Conforme con Koop, Pesaran y Potter (1996) y Pesaran y Shin (1998), es utilizado una función impulso-respuesta generalizada ya que esta es invariante al orden de variables consideradas en el VAR. El orden del rezago en el VAR fue escogido con base en el criterio de información de Hannan-Quinn.

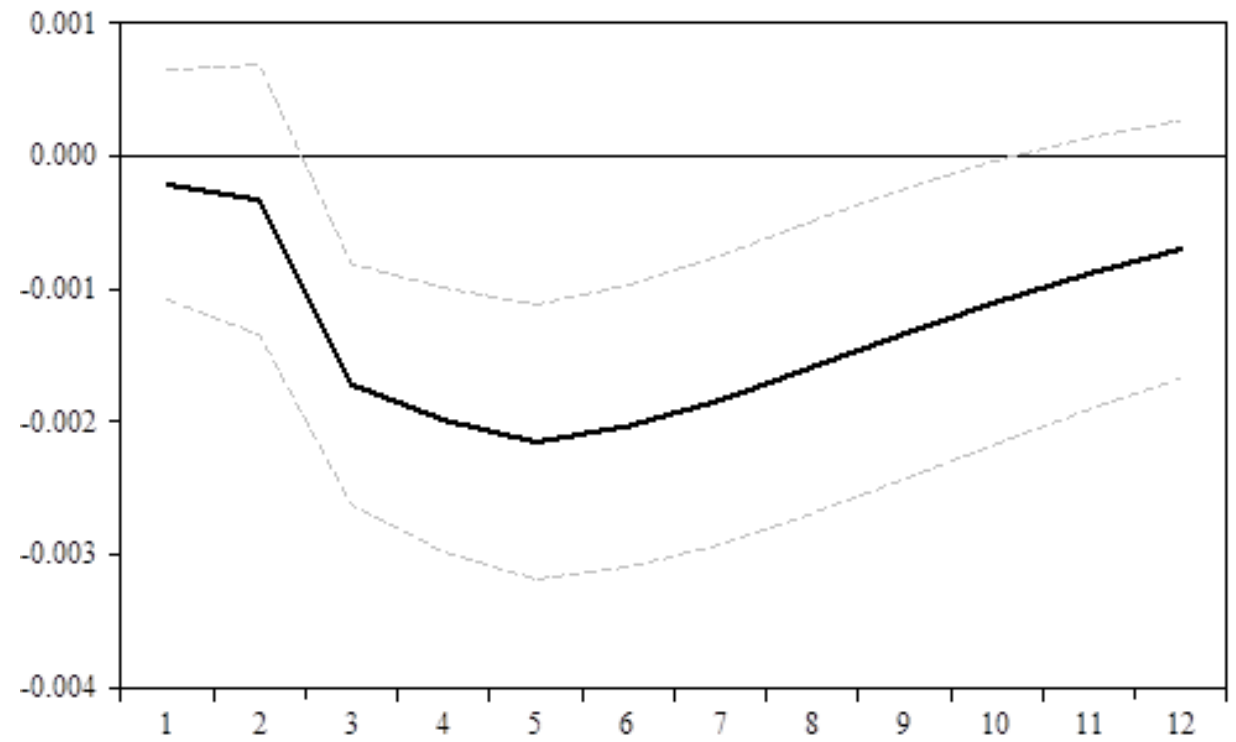

Figura 4. Respuesta acumulada del Error de pronóstico a la Comunicación del Banco Central

Nota: Respuesta acumulada a una innovación generalizada de una desviación estándar 2 S.E. Elaboración propia.

La figura 4 muestra los resultados de la función impulso-respuesta generalizada para un período de 12 meses. La gráfica sugiere que un choque positivo inesperado de la comunicación provoca una reducción significa en los errores de pronóstico a partir del segundo mes. Además, el impacto es significativo hasta el mes diez. En conclusión, la comunicación del Banco Central de Colombia contribuye para la disminución del sesgo de las expectativas en el mercado financiero.

Como un complemento al análisis impulso-respuesta de la figura anterior, se realiza a continuación un análisis de descomposición de varianza. En la tabla 2 se presenta la descomposición de varianza de los errores de pronóstico ante una innovación en la comunicación y una innovación en los errores de pronóstico. Conforme puede ser observado, una innovación en la comunicación del banco central da cuenta por casi el $1.5 \%$ de la variación de los errores de pronóstico en el período inicial. El impacto de la comunicación se incrementa de forma monótona y al final del mes 12 explica el 42,5\% de la varianza de los errores de pronóstico. En suma, la evidencia apunta a que la comunicación debe ser considerada al momento de analizar los errores de pronóstico de inflación en el mercado financiero. 
Revista Mexicana de Economía y Finanzas Nueva Época, Vol. 14 No. 4, pp. 617-631 DOI: https://doi.org/10.21919/remef.v14i4.322

Tabla 5. Descomposición de varianza de los errores de pronóstico

\begin{tabular}{|c|c|c|c|}
\hline Período & Error estándar & Error de Pronóstico & $\begin{array}{c}\text { Comunicación del } \\
\text { Banco Central }\end{array}$ \\
\hline 1 & 0.004464 & 100.0000 & 0.000000 \\
\hline 2 & 0.005240 & 99.85260 & 0.147395 \\
\hline 3 & 0.005850 & 92.17827 & 7.821731 \\
\hline 4 & 0.006282 & 83.75311 & 16.24689 \\
\hline 5 & 0.006674 & 75.52991 & 24.47009 \\
\hline 6 & 0.006985 & 69.34143 & 30.65857 \\
\hline 7 & 0.007224 & 64.91271 & 35.08729 \\
\hline 8 & 0.007397 & 61.92360 & 38.07640 \\
\hline 9 & 0.007518 & 59.95562 & 40.04438 \\
\hline 10 & 0.007598 & 58.69449 & 41.30551 \\
\hline 11 & 0.007651 & 57.90365 & 42.09635 \\
\hline 12 & 0.007684 & 57.41904 & 42.58096 \\
\hline
\end{tabular}

Nota: Elaboración propia.

\section{Conclusiones}

Este trabajo analizó la influencia de la comunicación del Banco Central de Colombia en los errores de pronóstico de inflación para la economía colombiana. Las expectativas de inflación fueron extraídas de los bonos de deuda pública y el error de pronóstico fue calculado con base en la diferencia entre las expectativas y la meta de inflación. Por otra parte, los efectos de la comunicación del banco central sobre el error de pronóstico fueron capturados por medio de los componentes inesperados en las decisiones de política monetaria. Luego de construidas las variables, fueron realizadas las estimaciones econométricas.

Los resultados encontrados permiten realizar las siguientes observaciones. Primero, la comunicación del Banco Central de Colombia es una herramienta que promueve la convergencia entre las expectativas del mercado financiero y las metas de inflación anunciadas por el banco central. En consecuencia, la comunicación muestra efectividad en la gestión de las expectativas.

Segundo, el análisis impulso respuesta muestra que la comunicación reduce el error de pronóstico y sus efectos son significativos por varios meses. Además, desde el análisis de descomposición de varianza, se constata que cerca de la mitad de la varianza del error de pronóstico es explicada como resultado de innovaciones de comunicación del banco central. Es decir, la evidencia presentada apunta a que existe un canal de las expectativas ya que el banco central muestra capacidad para hacer converger las expectativas hacia la meta de inflación.

Tercero, y último, existen otras variables no relacionadas con la comunicación que afectan el desanclaje de las expectativas de inflación. Una de ellas es la misma persistencia del error de pronóstico. Otros factores que contribuyen al error de pronóstico son el comportamiento del mercado cambiario, la incertidumbre global y la política monetaria de la Reserva Federal de Estados Unidos. Todos estos factores muestran efectos en el error de pronóstico y tienen capacidad de desalinear las expectativas del mercado con relación a la meta de inflación. 


\section{Referencias}

Alichi, A., Catão, L., Leigh, D., Pescatori, A., Kamenik, O., Kim, H., Laxton, D., Portillo, R., Simon, J., Zanna, F. (2011). World Economic Outlook (September). International Monetary Fund. Chapter $3,101-133$.

Apergis, N. (2017). New Evidence on the Ability of Asset Prices and Real Economic Activity Forecast Errors to Predict Inflation Forecast Errors. Journal of Forecasting, 36(5), 557-565.

Babecký, J., Podpiera, J. (2011). Inflation Forecast Errors in the Czech Republic Evidence from a Panel of Institutions. Eastern European Economics, 49(1), 72-83.

Bec, F., De Gaye, A. (2016). How do oil price forecast errors impact inflation forecast errors? An empirical analysis from US, French and UK inflation forecasts. Economic Modelling, 53(2), 75-88.

Bernanke, B., Laubach, T., Mishkin, F., Posen, A. (1999). Inflation Targeting: Lessons from the International Experience. Princeton University Press, Princeton, 382 p.

Blinder, A., Ehrmann, M., Fratzcher, M., De Haan, J., Jansen, D. (2008). Central Bank Communication and Monetary Policy. Journal of Economic Literature, 46(4), 910-945.

Byrne, J., Fiess, N. (2016). International capital ows to emerging markets: National and global determinants. Journal of International Money and Finance, 61(3), 82-100.

Capistrán, C., Timmermann, A. (2009). Disagreement and biases in inflation expectations. Journal of Money, Credit and Banking, 41(2-3), 365-396.

Christensen, I., Dion, F., Reid, C. (2004). Real return bonds, inflation expectations and the Break Even Inflation rate. Bank of Canada Working Paper No. 2004-43.

Connolly, E., Kohler, M. (2004). News and Interest Rate Expectations: A Study of Six Central Banks. En: Kent, K. and S. Guttman (eds.). The Future of Inflation Targeting, Reserve Bank of Australia, 108-134. Sydney.

Crowe, C. (2010). Testing the Transparency Benefits of Inflation Targeting: Evidence from Private Sector Forecasts. Journal of Monetary Economics, 57(2), 226-232.

De Pooter, M., Robitaille, P., Walker, I., Zdinak, M. (2014). Are Long-Term Inflation Expectations Well Anchored in Brazil, Chile and Mexico?. International Journal of Central Banking, 10(2): 337-400.

Demertzis, M., Marcellino, M., Viegi, N. (2009). Anchor for inflation expectations. De Nederlandsche Bank, Working paper No. 229.

Dovern, J., Fritsche, U., Slacalek, J. (2009). Disagreement among Forecasters in G7 Countries. ECB Working Paper, 1082.

Dräger, L., Lamla., M. (2012). Updating inflation expectations: evidence from micro-data.Economics Letters, 117(3), 807-810.

Ehrmann, M., Eijffinger, S., Fratzscher, M. (2012). The role of central bank transparency for guiding private sector forecasts. Scandinavian Journal of Economics, 114(3), 1018-1052.

Ehrmann, M., Fratzscher, M., Gürkaynak, R., Swanson, E. (2011). Convergence and Anchoring of Yield Curves in the Euro Area. The Review of Economics and Statistics, 93(1): 350-364.

Ellingsen, T., Söderström, U. (2001). Monetary Policy and Market Interest Rates. The American Economic Review, 91(5), 1594-1607.

Engle, R. (1982). Autoregressive conditional heteroscedasticity with estimates of the variance of United Kingdom inflation. Econometrica, 50(4): 987-1008.

Filardo, A., Guinigundo, D. (2008). Transparency and Communication in Monetary Policy: A Survey of Asian Central Banks. Paper presented during the BSP-BIS Joint High-Level Conference on Transparency and Communication in Monetary Policy on 1 February 2008 in Manila, Philippines.

Galvis, J., Anzoátegui, J. (2017). Announcements credibility and government securities: evidence from Colombia. Applied Economics Letters, 25(4), 278-282.

Galvis, J., De Moraes, C., Anzoátegui, J. (2017). Efectos de los anuncios de política monetaria sobre la volatilidad de la tasa de cambio: un análisis para Colombia, 2008-2015. Lecturas de Economía, 87 (julio-diciembre) pp. 67-95.

Geraats, P. (2002). Central Bank Transparency. The Economic Journal, 112(483), 532-65.

González-Rozada, M., Levy-Yeyati, M. (2008). Global Factors and Emerging Market Spreads. Economic Journal, 118(533), 1917-1936.

Gürkaynak, R., Levin, A., Swanson, E. (2010). Does Inflation Targeting Anchor Long-Run Inflation Expectations? Evidence from Long-Term Bond Yields in the U.S., U.K., and Sweden. Journal of the European Economic Association, 8(6), 1208-1242.

Gürkaynak, R., Sack, B., Swanson, E. (2005). Do Actions Speak Louder Than Words?. The Response of Asset Prices to Monetary Policy Actions and Statements. International Journal of Central Banking, $1(1), 55-93$.

Jansen, D., De Haan, D. (2007). The Importance of Being Vigilant: Has ECB Communication Influenced Euro Area Inflation Expectations?. CESifo Working Paper Series No. 2134: 1-29. Munich.

Johnson, D. (2003). The Effect of Inflation Targets on the Level of Expected Inflation in Five Countries. Review of Economics and Statistics, 85(4), 1076-81.

Koop, G., Pesaran, M. H., Potter, S. M. (1996). Impulse response analysis in non-linear multivariate models. Journal of Econometrics, 74(1), 119-147. 
Kurz, M. (2005). Diverse Beliefs, Forecast Errors and Central Bank Policy. Stanford Institute for Economic Policy Research. Working paper. 04-04.

Kuttner, N. (2001). Monetary Policy Surprises and Interest Rates: Evidence from the FED FUNDS Futures Market. Journal of Monetary Economics, 47(3), 523-544.

Levin, T. (2014). The design and communication of systematic monetary policy strategies. Journal of Economic Dynamics \& Control, 49(December), 52-69.

Mankiw, N., Reis, R., Wolfers, J. (2004). Disagreement about Inflation Expectations. NBER Macroeconomics Annual, Vol. 18: 209-268.

Martinez, D., Moreno, J., Rojas, J. (2015). Evolución de la relación entre bonos locales y externos del gobierno colombiano frente a choques de riesgo. Borradores de Economía, 919, 1-23.

Melo, L., Granados, J. (2010). Expectativas y prima por riesgo inflacionario bajo una medida de compensación a la inflación. Borradores de Economía, 589, 1-25.

Montes, G., Oliveira, L., Curi, A., Nicolay, R. (2016). Effects of transparency, monetary policy signalling and clarity of central bank communication on disagreement about inflation expectations. Applied Economics, 47(32), 1-18.

Nelson, D. (1991). Conditional Heteroskedasticity in Asset Returns: A new Approach. Econometrica, 59(2): 349-380.

Patton, A., Timmermann, A., (2010). Why do forecasters disagree? Lessons from the term structure of cross-sectional dispersion. Journal of Monetary Economics, 57(7), 803-820.

Pedersen, M. (2015). What affects the predictions of private forecasters? The role of central bank forecasts in Chile. International Journal of Forecasting, 31(4), 1043-1055.

Pesaran, M. H., Shin, Y. (1998). Generalized impulse response analysis in linear multivariate models. Economic Letters, 58(1), 17-29.

Rincón, H., Rodríguez, N. (2016). Pass-Through of Exchange Rate Shocks on Inflation: A Bayesian Smooth Transition VAR Approach. Borradores de Economía, No. 930, 1-75. Bogotá.

Scharnagl, M., Stapf, J. (2015). Inflation, deflation, and uncertainty: What drives euro-area option-implied inflation expectations, and are they still anchored in the sovereign debt crisis?. Economic Modelling, 48(C), 248-269

Siklos, P. (2008). Inflation Targeting Around the World. Emerging Markets Finance and Trade, 44(6), 17-37.

Söderlind, P. (2011). Ination risk premia and survey evidence on macroeconomic uncertainty. International Journal of Central Banking, 7(2), 113-133.

Tovar, J. (2017). On the rationality and efficiency of inflation forecasts: Evidence from advanced and emerging market economies. Research in International Business and Finance, 40(april), 175-189. 